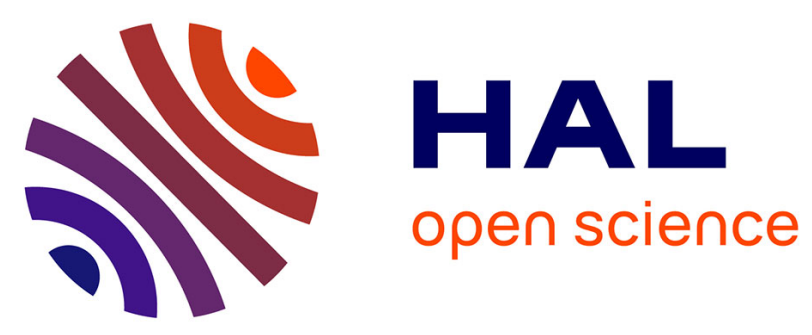

\title{
HIV-1 viral infectivity factor interacts with microtubule-associated protein light chain 3 and inhibits autophagy
}

Sophie Borel, Véronique Robert-Hebmann, Jamal Alfaisal, Ashish Jain, Mathias Faure, Lucile Espert, Laurent Chaloin, Jean-Christophe Paillart, Terje Johansen, Martine Biard-Piechaczyk

\section{To cite this version:}

Sophie Borel, Véronique Robert-Hebmann, Jamal Alfaisal, Ashish Jain, Mathias Faure, et al.. HIV1 viral infectivity factor interacts with microtubule-associated protein light chain 3 and inhibits autophagy. AIDS. Official journal of the international AIDS Society, 2015, 29 (3), pp.275-286. 10.1097/QAD.0000000000000554 . hal-02421817

\section{HAL Id: hal-02421817 https://hal.science/hal-02421817}

Submitted on 16 Nov 2020

HAL is a multi-disciplinary open access archive for the deposit and dissemination of scientific research documents, whether they are published or not. The documents may come from teaching and research institutions in France or abroad, or from public or private research centers.
L'archive ouverte pluridisciplinaire HAL, est destinée au dépôt et à la diffusion de documents scientifiques de niveau recherche, publiés ou non, émanant des établissements d'enseignement et de recherche français ou étrangers, des laboratoires publics ou privés. 
HIV-1 Vif interacts with LC3 and inhibits autophagy

Short title: Vif blocks autophagy

\section{Authors:}

Sophie BOREL ${ }^{1}$, Véronique ROBERT-HEBMANN ${ }^{1}$, Jamal ALFAISAL ${ }^{1}$, Ashish JAIN ${ }^{2}$, Mathias FAURE ${ }^{3,4,5,6,7}$, Lucile ESPERT ${ }^{1}$, Laurent CHALOIN ${ }^{1}$, Jean-Christophe PAILLART ${ }^{8}$, Terje JOHANSEN ${ }^{2}$, Martine BIARDPIECHACZYK ${ }^{1}$

\section{Affiliations:}

${ }^{1}$ Centre d'études d'agents Pathogènes et Biotechnologies pour la Santé (CPBS), Centre National de la Recherche Scientifique (CNRS) and Université de Montpellier, Montpellier, France.

${ }^{2}$ Molecular Cancer Research Group, University of Troms $\varnothing$ - The Arctic University of Norway, 9037 Troms $\varnothing$, Norway.

${ }^{3} \mathrm{CIRI}$, International Center for Infectiology Research, Université de Lyon, Lyon, France.

${ }^{4}$ Inserm, U1111, Lyon, France.

${ }^{5}$ Ecole Normale Supérieure de Lyon, Lyon, France.

${ }^{6}$ Université Lyon 1, Centre International de Recherche en Infectiologie, Lyon, France.

${ }^{7}$ CNRS, UMR5308, Lyon, France.3. INSERM U851 and Université de Lyon, Lyon, France

${ }^{8}$ Architecture et Réactivité de l'ARN, CNRS, Université de Strasbourg, Institut de Biologie Moléculaire et Cellulaire, Strasbourg, France.

Number of words in the abstract: 249

Number of words in the text: 3892

\section{Corresponding author:}

Martine Biard-Piechaczyk 
CPBS, CNRS UMR5236

1919 Route de Mende

34293 Montpellier Cedex 5

France

e-mail : martine.biard@cpbs.cnrs.fr

Telephone number: +33434359455

Fax number: +33434359411

\section{Support:}

Jamal Alfaisal is benefiting from the Dunia Beam Erasmus Mundus scholarship, funded with the support of the European Commission. Ashish Jain post-doctoral fellowship is funded by the Norwegian Research Council. HeLa A3G (Hela-APOBEC3G, \#9907), anti-A3G Ab (\#9906), anti-Vif Ab (clone 319, \#6459) and pNL4.3 (pNL 4-3, \#114) were obtained through the AIDS Research and Reference Program, Division of AIDS, NIAID, NIH. We thank J.M. Peloponese and A. Valenzuela Fernández for providing pNL4.3AVif and pcDNA-A3G, respectively and F.P. Blanchet for helpful scientific discussions and careful critical reading of the manuscript. 


\begin{abstract}
Objective: Autophagy, an important anti-viral process triggered during HIV-1 entry by gp41dependent membrane fusion, is repressed in infected CD4 T cells by an unknown mechanism. The aim of this study was to identify the role of Vif in the autophagy blockade.
\end{abstract}

Design/methods: To determine the role of Vif in autophagy inhibition, we used cell lines that express CD4 and CXCR4 and primary CD4 T cells. Pull-down experiments, immunoprecipitation assays and computational analyses were performed to analyze the interaction between Vif and LC3B, a major autophagy component, in presence or absence of the anti-viral host factor APOBEC3G, after HIV-1 infection or ectopic expression of Vif. Autophagy was analyzed after infection by viruses expressing Vif (NL4.3) or not (NL4.3 $\Delta$ Vif), or after exogenous Vif expression.

Results: We demonstrate that the C-terminal part of Vif interacts directly with LC3B, independently of the presence of APOBEC3G. Vif binds to pro-LC3 and Atg4-cleaved LC3 forms, and glycine 120, the amino acid conjugated to phosphatidylethanolamine on autophagosomes, is required. Importantly, we evidence that Vif inhibits autophagy during HIV-1 infection. Indeed, autophagy is detected in target cells infected by NL4.3 $\Delta$ Vif, but prevented in cells infected by NL4.3. Furthermore, autophagy triggered in NL4.3 $\Delta$ Vif-infected cells is inhibited when Vif is expressed in trans but is still active when target cells express a mutant of Vif that binds weakly to LC3B.

Conclusions: Our study unveils that Vif inhibits autophagy independently of its action on APOBEC3G and therefore suggest a new function of this viral protein in restricting innate anti-viral mechanisms.

\title{
Key words:
}

HIV, Vif, autophagy, LC3, APOBEC3G 


\section{Introduction}

Autophagy is a degradation process involving autophagy-related (Atg) proteins by which portions of cytosol are sequestered into double-membrane vesicles, namely autophagosomes, and delivered into lysosomes for degradation. Conjugation of Atg8 to phosphatidylethanolamine (PE) is central in this process [1, 2]. Mammals have six Atg8-like proteins (mAtg8s) that comprise three microtubuleassociated protein 1 light chain 3 (LC3) proteins (LC3A, B, and C), one gamma-aminobutyrate receptor-associated protein (GABARAP) and two GABARAP-like proteins (GABARAPL1 and GATE16/GABARAPL2) [3]. These proteins are synthesized as precursors that are rapidly processed by Atg4, leading to exposure of a glycine residue that can be conjugated to PE [4]. Besides its role in homeostasis, autophagy plays an important role in the innate and adaptive immune responses against pathogens [5, 6]. Particularly, HIV-1 envelope triggers autophagy in bystander CD4 T cells, which leads to their apoptosis, a mechanism now recognized as central to immunodeficiency [7]. In contrast, when CD4 T cells are productively infected, autophagy is repressed [8], strongly suggesting that one or several viral proteins are involved in this autophagy inhibition. Among them, Vif (Viral infectivity factor) favors HIV-1 infection by degrading the anti-viral host factors APOBEC3s (Apolipoprotein B mRNA-editing enzyme-catalytic polypeptide-like 3), and especially APOBEC3G (A3G) [9-12]. Vif binds to A3G and an E3 ubiquitin ligase complex composed of ElonginB (EloB), ElonginC (EloC), Cullin 5 (Cul5), Rbx2 and CBF- $\beta$ to trigger A3G degradation by the ubiquitin-proteasome system (UPS) [13-15]. The N-terminus of Vif interacts with A3G and CBF- $\beta$ while a zinc-binding motif $(\mathrm{HCCH})$ and a SOCS box-like domain in the C-terminal part bind to components of the E3 ligase complex [16, 17], Cul5 [18] and EloC $[13,19]$, respectively. Furthermore, the 161-PPLP-164 motif plays an important role in Vif function and infectivity [20-24].

We demonstrate here that Vif neo-synthesized in HIV-1-infected CD4 T cells is involved in autophagy blockage. This inhibition requires the C-terminal part of Vif, which binds directly to LC3B independently of the presence of A3G. These results reveal an additional important pro-viral function of Vif through autophagy inhibition. 


\section{Methods}

Cells and transfection. Adherent and CD4 T cells were cultured in DMEM and RPMI, respectively, supplemented with $1 \%$ penicillin/streptomycin and 10\% FCS. Primary CD4 T cells from blood of healthy human donors provided by EFS (Etablissement Français du Sang, France) were purified by negative selection (StemCell Technologies) and stimulated with $2 \mu \mathrm{g}$ phytohemagglutinin (SigmaAldrich) and $100 \mathrm{U}$ interleukin-2 (Boehringer Mannheim) per $\mathrm{mL}$ for 3 days before their infection. Procedures using human cells were approved by the Human Experimentation and Ethics Committee of the CNRS. . Starvation was triggered by cell culture in EBSS (Gibco) at $37^{\circ} \mathrm{C}$ for $4 \mathrm{~h}$. Adherent cells were transfected using Turbofect (Fermentas GMBH) according to the manufacturer's instructions.

Plasmids and Gateway cloning. Plasmid constructs were made using both traditional cloning and Gateway recombination cloning system (Invitrogen). Mutagenesis of Vif and LC3 genes was performed using the QuickChange II site-Directed Mutagenesis Kit (Agilent technology).

GST pull-down experiments. GST pull-down assays were performed by incubating immobilized GST or GST-tagged human ATG8 proteins with ${ }^{35} \mathrm{~S}$-labeled in vitro translated Vif fused to the Myc tag. ${ }^{35} \mathrm{~S}$ labeled Myc-Vif was produced using the TNT T7 Quick Coupled Transcription/Translation System (Promega) in the presence of $\left.{ }^{35} \mathrm{~S}\right]$ methionine (Amersham Biosciences) and pre-cleared by incubating with glutathione-coupled sepharose beads (GE healthcare) for 30min prior to incubation with immobilized GST or GST fusion proteins for at least $2 \mathrm{~h}$ at $4^{\circ} \mathrm{C}$. Unbound proteins were removed by washings. The proteins were then eluted by boiling for $5 \mathrm{~min}$ in SDS gel loading buffer and separated by SDS-PAGE. ${ }^{35}$ S-labeled proteins were detected on a Fujifilm bioimaging analyzer BAS-5000 (Fuji). Recombinant proteins were detected by Coomassie blue staining (CBB).

GST pull-down experiments were also performed ex vivo. Since Vif is known to be rapidly degraded by UPS $[25,26]$, MG132 was added to the cells at $20 \mu \mathrm{M}$ for $12 \mathrm{~h}$ before lysis. Cell lysis was done $48 \mathrm{~h}$ 
post-transfection. The lysates were incubated with glutathione-coupled sepharose beads for $3 \mathrm{~h}$ at $4^{\circ} \mathrm{C}$. Unbound proteins were removed by washings. Bound proteins were then eluted by boiling for 10 min in SDS gel loading buffer and separated by gel electrophoresis.

Co-immunoprecipitation assays. Immunoprecipitation of LC3 was performed using protein Asepharose beads for $2 \mathrm{~h}$ at $4^{\circ} \mathrm{C}$. After washings, bound proteins were eluted as described above. Interaction between transfected 3xFlag-Vif and endogenous LC3 was also performed using EZview red anti-Flag M2 antibody (Sigma-Aldrich). 48h post-transfection, E64d and pepstatin A were added for 3h at $10 \mu \mathrm{g} / \mathrm{mL}$ each and cells were then lysed. Lysates were incubated with beads for $2 \mathrm{~h}$ at $4^{\circ} \mathrm{C}$. After washings, bound proteins were eluted as described above.

Western blotting. Cell lysates were electrophoresed in $10 \%$ Prosieve $^{\mathrm{TM}} 50$ gel (Lonza), transferred to PVDF membranes and western-blotting was done with indicated antibodies. Quantification of protein expression was performed by densitometry analysis using the Genesys software (Syngene).

HIV-1 infections. HEK cells were transfected with pNL4.3WT or pNL4.3AVif and supernatants were collected two days later. HIV-1 infections were then performed with normalized amounts of supernatants (1 MOI). HEK-CD4-CXCR4 cells were also infected by co-culture with CD4 T cells infected by NL4.3WT or NL4.3AVif (same percentage of infected cells, at least $50 \%$ ), or with chronically infected MOLTX4 or $8 . E 5$ cells, as previously described [8], and Jurkat cells by co-culture with HEK cells transfected with pNL4.3WT or pNL4.3AVif. The percentage of infected cells was analyzed using the HIV KC57 FITC kit (Beckman Coulter).

Fluorescence microscopy studies. HEK cells were transfected with pEGFP-LC3 plasmid and fixed two days later by $3.7 \%$ paraformaldehyde in PBS for $10 \mathrm{~min}$. Autophagic cells are defined as cells containing at least 5 LC3 positive spots. 
For detection of endogenous LC3, cells were fixed in a $1: 1$ acetone/methanol for $5 \mathrm{~min}$ at $-20^{\circ} \mathrm{C}$. Cells were then incubated with the rabbit anti-LC3 Ab (and with the mouse anti-p24 Ab when autophagy is analyzed in HIV-infected cells) for $1 \mathrm{~h}$, washed, and then incubated with corresponding secondary antibody(ies) for 30min. Nuclei were labeled with Dapi and cells were examined by epifluorescence using a Leica microscope. The average number of LC3 dots was evaluated in the untreated condition for each cell type to define the optimal threshold. Autophagic primary CD4 T cells were defined as cells containing more than 3 LC3 dots, and autophagic HEK cells or CD4 T cell lines (Jurkat cells and H9 cells) as cells containing at least 8 LC3 dots. More than 100 transfected cells were analyzed by 3 investigators. Results are from at least 3 independent blind experiments.

Computational analysis. The recently solved crystal structure of Vif in complex with CBF$\beta /$ Cul5/EloB/EloC [27] was used for docking experiments (pdb 4N9F). Vif was extracted from the complex and refined by energy minimization in explicit water using the Charmm force field and NAMD 2.9 program for 50,000 steps of conjugate gradient. The crystal structure of LC3 was taken from the LC3-p62 complex [28] solved at $1.56 \AA$ (pdb code 2ZID). For rigid docking, the Cluspro 2.0 [29] server (http://cluspro.bu.edu/) was used without including any attractive or repulsive constraints.

Statistics. Variance analysis of the percentage of autophagic cells obtained after infection by NL4.3WT versus NL4.3DVif was performed after arc sine transformation of the data [30]. Differences were considered significant at $* P<0.05, * * P<0.01$, and ${ }^{* * *} P<0.001$.

\section{Results}

\section{Vif binds to LC3 independently of A3G}

We first demonstrated in vitro that Vif interacted with the human ATG8 family members (Fig. 1a). Interaction between Vif and three members of the LC3 family was then confirmed within cells (Fig. 
1b). As LC3B is the ATG8 protein characterized as an autophagosome marker per se, we next focused our research on this Atg, hereafter referred to as LC3. We further demonstrated the interaction between endogenous Vif and LC3 during productive infection of primary CD4 T cells (Fig. 1c). Since primary CD4 T cells express A3G, this result suggests that A3G does not impede Vif binding to LC3. To verify this important point, we studied the binding between Vif and LC3 in presence of ectopically expressed A3G and confirmed that A3G does not prevent this interaction (Fig. 1d). Interestingly, we observed that A3G was also co-precipitated with Vif, suggesting that the binding interfaces of Vif for LC3 and A3G are not mutually exclusive.

\section{The C-terminal part of Vif interacts with LC3}

Proteins that interact with LC3 generally harbor a LC3-interacting region (LIR) motif characterized by a $\mathrm{W} / \mathrm{F} / \mathrm{Yxxl} / \mathrm{L} / \mathrm{V}$ sequence $[31,32]$. By computational analysis, we found the presence of three potential LIR motifs, highlighted within both the sequence and the crystal structure of Vif (Fig. 2a). To determine their functional significance, we generated Vif mutants in which the first amino acid of each putative LIR sequence was replaced by an alanine, a type of LIR mutation already known to abolish the interaction with LC3 [31]. We tested single, double (not shown), and triple mutants, and demonstrated that the putative LIR motifs are not involved in the interaction with LC3 (Fig. 2b). To get an overview of the Vif-LC3 interaction and to determine the domains involved, we used fastFourier transform-based rigid docking. By this theoretical approach, we obtained one main populated model for the Vif-LC3 complex (Fig. 2c). In this model, LC3 binds to the smaller C-domain ( $\alpha$-domain) of Vif, encompassing the $\mathrm{HCCH}$ and SOCS motifs, mainly by hydrophobic contacts. An interaction between the C-terminal part of Vif and G120 of LC3 was shown to be important for the complex formation. To evaluate the importance of each sub-domain (HCCH and SOCS) in LC3 binding, a rigid docking prediction was performed using the truncated form of Vif (1-143) in which the SOCS motif has been deleted. Surprisingly, a similar binding of Vif to LC3 was observed when a large motion of LC3 (about $30^{\circ}$ ) was integrated. The missing SOCS box of Vif, however, led to a smaller 
number of interactions between both proteins suggesting a weaker interaction (Fig. 2d). A larger deletion of the C-terminal of Vif leading to the loss of $\mathrm{HCCH}$ motif renders the $\alpha$-helix of the SOCS box too flexible and could unfortunately not result in accurate binding predictions (data not shown). Altogether, the predictions suggested that the C-terminal part of Vif starting from the zinc-finger and up to the SOCS box-like motif is involved in LC3 binding.

To confirm this hypothesis, several mutants were constructed: Vif1-159, Vif1-143 and Vif1-99 where the oligomerization domain (161-PPLP-164), the SOCS box-like motif and the HCCH motif were deleted, respectively (Fig. 2e). As shown in figure 2f, Vif1-159 still bound to LC3 whereas Vif1-143 and Vif1-99 had mainly lost their ability to interact with LC3, indicating that the domain 144-159, containing the SOCS box-like motif, is important for this interaction.

\section{Vif binds to pro-LC3 and Atg4-cleaved LC3, and the glycine 120 of LC3 is important for Vif interaction}

To determine which form(s) of LC3 can bind to Vif, we tested different LC3 constructs. We first analyzed a pro-LC3 mutated on two amino acids required for processing, F80 and L82 (proLC3F80A/L82A) (Fig. 3a), and showed that this mutant still bound to Vif (Fig. 3b), indicating that Vif interacts with the pro-LC3 independently of the FxL motif [33]. Next, we wondered whether Vif could bind to LC3 1-120 that comprises LC3-I and LC3-II and LC3 1-119 (Fig. 3c). As shown in figure 3d, Vif bound to LC3 1-120 but not to LC3 1-119, confirming that G120 is critical for the interaction with Vif and that Vif may bind to both LC3-I and LC3-II forms.

To determine whether Vif binds to one specific Atg4-cleaved form of LC3 or both LC3-I and LC3-II forms in the presence of A3G, pull-down studies were performed in cells stably expressing A3G in conditions that induce, or not, the formation of autophagosomes. We observed that both LC3-I and LC3-II were pulled-down by Vif but not by Vif1-99, still demonstrating that the C-terminal part of Vif is responsible for the interaction (Fig. 3e). The interaction of Vif with LC3-I is stronger than with LC3- 
II, suggesting that Vif does have a preference to LC3-I. This also confirms that the binding of A3G to Vif does not affect LC3 binding to Vif.

\section{Neo-synthesized Vif inhibits autophagy during HIV-1 infection}

We and others previously reported that autophagy is blocked in CD4 T cells that have been productively infected by HIV-1 $[8,34]$, but the actors of this inhibition have not yet been identified. The interaction between Vif and LC3 suggests that Vif may act directly on autophagy. We have thus analyzed the level of autophagy in different types of cells after their infection by the HIV-1 strain NL4.3 that expresses Vif (NL4.3WT) or not (NL4.3AVif). Autophagy was first analyzed by following the formation of autophagosomes [35] in Jurkat cells, which do not express A3G, and H9 cells, which express A3G, after 4 days of infection. The percentage of autophagic cells was higher in cells infected by NL4.3AVif compared to cells infected by NL4.3WT (Fig. 4a,b), indicating that Vif dramatically decreases the number of autophagosomes independently of the presence of A3G. We confirmed these data in primary CD4 T cells (Fig.4c). Same results were also obtained in HEK cells stably expressing CD4 and CXCR4 (HEK-CD4-CXCR4) after their infection by free NL4.3WT or NL4.3AVif (Fig. $5 a)$.

To study the role of Vif on the autophagic flux, we blocked the autophagosome-lysosome fusion with chloroquine. Autophagy was still higher in primary CD4 T cells (Fig. 4d), demonstrating that Vif acts on the first steps of autophagy. We also analyzed autophagy in H9 cells at longer times of infection, in presence or absence of chloroquine. Autophagy was also higher in cells infected for 11 days by NL4.3AVif compared to cells infected by NL4.3WT, in presence or absence of chloroquine (Supplemental Fig.1).

By western blot, we also demonstrated that LC3-II expression level is higher in Jurkat cells infected by free NL4.3AVif than by NL4.3WT (Supplemental Fig. 2a) and similar results were obtained in HEK cells transfected with pNL4.3 $\Delta$ Vif or pNL4.3WT (Supplemental Fig. 2b). 
Moreover, ectopic expression of Vif dramatically decreased autophagy induced by Torin, demonstrating that Vif alone is able to block induced autophagy (Supplemental Fig. 3a). We confirmed by western blot experiments that ectopic Vif inhibits the autophagy pathway (Supplemental Fig. 3b).

Furthermore, Vif inhibits the formation of autophagosomes independently of the way of infection (co-culture or free virus) since the same result was obtained in HEK-CD4-CXCR4 infected by coculture with CD4 T cells infected by NL4.3WT or NL4.3AVif (Fig. 4e, two upper panels). To determine whether Vif coming from the virus $[36,37]$ and/or neo-synthesized Vif [38] upon replication act on autophagy, HEK-CD4-CXCR4 cells were infected by co-culture with chronically infected CD4 T cells expressing WT HIV-1 (MoltX4 cells) or 8E5 cells that carry a single copy of the HIV genome point-mutated for reverse transcriptase. In both cases, HIV-1 can enter HEK-CD4-CXCR4 target cells and incoming Vif is present, but Vif is only produced in target cells infected by the WT virus, not the one produced from 8E5 cells. Interestingly, autophagy was only observed in HEK-CD4CXCR4 cells co-cultured with 8 E5 cells and cells infected by NL4.3 $\Delta$ Vif (Fig. $4 \mathrm{e}$, two bottom panels), demonstrating that neo-synthesized Vif is responsible for autophagy blockade. Furthermore, as Vif inhibits autophagy independently of the presence of A3G, we analyzed its action on p24 production in permissive cells. The percentage of cells infected by either NL4.3WT or NL4.3 $\Delta$ Vif was equivalent (Supplemental Fig. 4a) but the intracellular expression level of p24 was increased in cells infected by NL4.3WT (Supplemental Fig. 4b,c). The fact that Vif both decreases autophagy and increases the intracellular production of p24 in infected permissive cells strongly suggests that the inhibitory function of Vif on autophagy promotes viral replication independently of A3G degradation by Vif.

\section{The Vif C-terminal domain is responsible for inhibition of autophagy}

To analyze whether Vif inhibits autophagy through its C-terminal domain, which directly interacts with LC3, we studied the formation of autophagosomes in NL4.3 $\Delta$ Vif-infected cells after expression in trans of WT Vif or Vif1-99 that has largely lost its ability to interact with LC3. Infections by NL4.3WT were 
performed in parallel. Controls have been done without Vif complementation (Fig. 5a). Autophagy was down-regulated only when cells infected by NL4.3AVif were complemented in trans with WT Vif (Fig.5b) but not with Vif1-99 (Fig. 5c), indicating that the C-terminal region of Vif, which binds LC3, is required to inhibit autophagy.

\section{Discussion}

Despite its small size, Vif is used as a binding platform to interact with numerous partners. The Nterminus of Vif possesses a well-defined topology and contains several motifs that interact with various human A3 proteins [39-43], RNAs [20] and CBF- $\beta[14,15]$. On the contrary, the unbound Cterminal part has a disordered nature, usually used to mediate sequential/differential binding with multiple partners $[44,45]$. We demonstrate here that the SOCS box-like motif present in the Cterminus of Vif interacts with LC3 in a LIR-independent manner [46]. Surprisingly, a putative LIR, called LIR3 (YLAL), is located within this sequence, but mutation of Y147 to A does not inhibit Vif binding to LC3. Very recently, a non-canonical LIR motif, termed CLIR, which does not contain the first aromatic residue and preferentially binds to LC3C, was identified in the sequence of NDP52 [47], an ubiquitin-binding autophagy receptor [48]. As Vif also interacts with the other members of the mAtg8 family, including LC3C (Fig. 1b), our result suggests that Vif may interfere with the physiological roles of these proteins through a non-canonical LIR motif in the SOCS box-like domain. Further investigation will be needed to study the role as well as the precise mode of the interaction between Vif and other members of Atg8 family proteins. The SOCS box-like domain binds to EloC, suggesting that EloC and LC3 may compete for binding to Vif. This was confirmed by docking experiments showing that LC3 could not bind anymore to the SOCS box-like motif when EloC is bound to Vif (Supplemental Fig. 5). However, the expression level of A3G was not significantly modified upon over-expression of LC3 (data not shown), strongly suggesting that even if a competition exists between LC3 and EloC for binding to Vif, the concentration of Vif in infected cells is sufficient to allow both A3G degradation and inhibition of autophagy through LC3 binding. This 
hypothesis is reinforced by the fact that only neo-synthesized Vif can repress autophagy. Indeed, only few Vif molecules are brought by the virus during its entry into target cells whereas Vif is well expressed in the cytoplasm of infected cells [49], before its degradation by the UPS [25, 26]. Several viral proteins have already been shown to inhibit autophagy and to favor viral replication through a direct interaction with Atgs, and Beclin 1 was described as the main target [50]. Moreover, Kyei and collaborators were the first group to show, in myeloid U937 cells, that LC3 is present in complexes with HIV Gag [51]. This interaction might be indirect through LC3 as Vif can bind to Gag. We currently do not know whether LC3 targeting is a peculiar strategy of HIV to control autophagy or a more common way of autophagy control by viruses. However, we have discovered a new function of Vif to promote viral replication independently of the presence of A3G. Of note, besides its interaction with A3G, it has been shown recently that Vif is able to pull-down AMBRA1 and p62, proteins involved in autophagy [14], but the consequences of these interactions are yet unknown. We could not see a significant direct interaction between recombinant p62 and in vitro translated Vif whereas both proteins were co-immunoprecipitated from cells, suggesting an indirect interaction (data not shown). This indirect interaction may involve LC3B, but further investigation will be required to answer this point.

In cells, pro-LC3 is rapidly processed, suggesting that even if Vif can bind to pro-LC3, it may act essentially on the Atg4-cleaved form of LC3, i.e. LC3-I and LC3-II. In addition, our results highlight the role of G120 of LC3 in the interaction with Vif, a crucial amino acid responsible for the conjugation to PE [2]. Vif can thus inhibit the formation of autophagosomes by acting all along the LC3-dependent ubiquitin-like conjugation system, but the precise mechanism is still unclear. No major degradation of LC3 was observed after binding to Vif (data not shown), ruling out the possibility that LC3, as A3G, could be degraded by UPS after Vif binding.

We previously demonstrated that autophagy is triggered in CD4 T cells by gp41-dependent membrane fusion $[7,52]$, and that autophagy is inhibited when these target cells are productively infected [8]. Now, our data show that Vif, expressed in productively infected cells, is responsible for 
the inhibition of the formation of the autophagosomes after binding to LC3 $[53,54]$. This also explains why autophagy is uncontrolled in bystander CD4 T cells after their contact with HIV-1infected cells. Indeed, in these non-activated cells, HIV-1 can enter but its genome is not integrated into the host cell genome and thus no viral protein can be synthesized. The role of Vif in the blockade of autophagy is strengthened by the fact that Vif can also block autophagy induced by Torin when produced ectopically in the cells. However, we cannot exclude a role of other viral proteins in the inhibition of autophagy all along the viral life cycle, and especially Nef that was already shown to control autophagy by binding to IRGM [55] and Beclin 1 [51] in macrophages. One hypothesis might be that both Nef and Vif « work in concert » to inhibit autophagy by interacting with several, different Atgs in CD4 T cells.

Taken together, our results demonstrate a novel and important function of Vif to constrain the antiviral mechanisms used by the cells to eliminate HIV-1, independently of its role in A3G degradation.

\section{Acknowledgements}

S.B., V.R-H, J.A. and A.J. performed experiments and analyzed data; M.F. and L.E. contributed to study design, L.C. performed computational analysis, J-C.P and T.J. contributed to study design and M.B-P contributed to study design and wrote the article. This work was supported by institutional funds from the Centre National de la Recherche Scientifique (CNRS) and the Montpellier University and grants from the Agence Nationale de Recherches sur le SIDA et les Hépatites Virales (ANRS) to Martine Biard-Piechaczyk, from Sidaction to Lucile Espert, and from the Norwegian Research Council to Terje Johansen.

\section{References}

1. Ichimura $Y$, Kirisako $T$, Takao $T$, Satomi $Y$, Shimonishi $Y$, Ishihara $N$, et al. A ubiquitin-like system mediates protein lipidation. Nature 2000,408:488-492.

2. Kabeya Y, Mizushima N, Ueno T, Yamamoto A, Kirisako T, Noda T, et al. LC3, a mammalian homologue of yeast Apg8p, is localized in autophagosome membranes after processing. Embo J 2000,19:5720-5728.

3. Shpilka T, Weidberg H, Pietrokovski S, Elazar Z. Atg8: an autophagy-related ubiquitin-like protein family. Genome Biol 2011,12:226. 
4. Tanida I, Sou YS, Ezaki J, Minematsu-Ikeguchi N, Ueno T, Kominami E.

HsAtg4B/HsApg4B/autophagin-1 cleaves the carboxyl termini of three human Atg8 homologues and delipidates microtubule-associated protein light chain 3- and GABAA receptor-associated protein-phospholipid conjugates. J Biol Chem 2004,279:36268-36276.

5. Deretic V, Levine B. Autophagy, immunity, and microbial adaptations. Cell Host Microbe 2009,5:527-549.

6. Levine B, Mizushima N, Virgin HW. Autophagy in immunity and inflammation. Nature 2011,469:323-335.

7. Espert L, Denizot M, Grimaldi M, Robert-Hebmann V, Gay B, Varbanov M, et al. Autophagy is involved in T cell death after binding of HIV-1 envelope proteins to CXCR4. J Clin Invest 2006,116:2161-2172.

8. Espert L, Varbanov M, Robert-Hebmann V, Sagnier S, Robbins I, Sanchez F, et al. Differential role of autophagy in CD4 T cells and macrophages during X4 and R5 HIV-1 infection. PLOS ONE 2009,4:e5787.

9. Lecossier D, Bouchonnet F, Clavel F, Hance AJ. Hypermutation of HIV-1 DNA in the absence of the Vif protein. Science 2003,300:1112.

10. Mariani R, Chen D, Schrofelbauer B, Navarro F, Konig R, Bollman B, et al. Species-specific exclusion of APOBEC3G from HIV-1 virions by Vif. Cell 2003,114:21-31.

11. Sheehy AM, Gaddis NC, Malim MH. The antiretroviral enzyme APOBEC3G is degraded by the proteasome in response to HIV-1 Vif. Nat Med 2003,9:1404-1407.

12. Chaipan C, Smith JL, Hu WS, Pathak VK. APOBEC3G restricts HIV-1 to a greater extent than APOBEC $3 F$ and APOBEC3DE in human primary CD4+ T cells and macrophages. J Virol 2013,87:444-453.

13. Bergeron JR, Huthoff $H$, Veselkov DA, Beavil RL, Simpson PJ, Matthews SJ, et al. The SOCS-box of HIV-1 Vif interacts with ElonginBC by induced-folding to recruit its Cul5-containing ubiquitin ligase complex. PLoS Pathog 2010,6:e1000925.

14. Jager S, Kim DY, Hultquist JF, Shindo K, LaRue RS, Kwon E, et al. Vif hijacks CBF-beta to degrade APOBEC3G and promote HIV-1 infection. Nature 2011,481:371-375.

15. Zhang W, Du J, Evans SL, Yu Y, Yu XF. T-cell differentiation factor CBF-beta regulates HIV-1 Vif-mediated evasion of host restriction. Nature 2011,481:376-379.

16. Henriet S, Mercenne G, Bernacchi S, Paillart JC, Marquet R. Tumultuous relationship between the human immunodeficiency virus type 1 viral infectivity factor (Vif) and the human APOBEC-3G and APOBEC-3F restriction factors. Microbiol Mol Biol Rev 2009,73:211-232.

17. Wolfe LS, Stanley BJ, Liu C, Eliason WK, Xiong Y. Dissection of the HIV Vif interaction with human E3 ubiquitin ligase. J Virol 2010,84:7135-7139.

18. Luo K, Xiao Z, Ehrlich E, Yu Y, Liu B, Zheng S, et al. Primate lentiviral virion infectivity factors are substrate receptors that assemble with cullin 5-E3 ligase through a $\mathrm{HCCH}$ motif to suppress APOBEC3G. Proc Natl Acad Sci U S A 2005,102:11444-11449.

19. Stanley BJ, Ehrlich ES, Short L, Yu Y, Xiao Z, Yu XF, et al. Structural insight into the human immunodeficiency virus Vif SOCS box and its role in human E3 ubiquitin ligase assembly. $J$ Virol 2008,82:8656-8663.

20. Bernacchi S, Mercenne G, Tournaire C, Marquet R, Paillart JC. Importance of the proline-rich multimerization domain on the oligomerization and nucleic acid binding properties of HIV-1 Vif. Nucleic Acids Res 2011,39:2404-2415.

21. Simon JH, Fouchier RA, Southerling TE, Guerra CB, Grant CK, Malim MH. The Vif and Gag proteins of human immunodeficiency virus type 1 colocalize in infected human $T$ cells. $J$. Virol. 1997,71:5259-5267.

22. Yang B, Gao L, Li L, Lu Z, Fan X, Patel CA, et al. Potent suppression of viral infectivity by the peptides that inhibit multimerization of human immunodeficiency virus type 1 (HIV-1) Vif proteins. J Biol Chem 2003,278:6596-6602.

23. Yang $\mathrm{S}$, Sun $\mathrm{Y}$, Zhang $\mathrm{H}$. The multimerization of human immunodeficiency virus type I Vif protein: a requirement for Vif function in the viral life cycle. J Biol Chem 2001,276:4889-4893. 
24. Donahue JP, Vetter ML, Mukhtar NA, D'Aquila RT. The HIV-1 Vif PPLP motif is necessary for human APOBEC3G binding and degradation. Virology 2008,377:49-53.

25. Fujita M, Akari H, Sakurai A, Yoshida A, Chiba T, Tanaka K, et al. Expression of HIV-1 accessory protein Vif is controlled uniquely to be low and optimal by proteasome degradation. Microbes Infect 2004,6:791-798.

26. Izumi T, Takaori-Kondo A, Shirakawa $\mathrm{K}$, Higashitsuji $\mathrm{H}$, Itoh $\mathrm{K}$, lo $\mathrm{K}$, et al. MDM2 is a novel E3 ligase for HIV-1 Vif. Retrovirology 2009,6:1.

27. Guo Y, Dong L, Qiu X, Wang Y, Zhang B, Liu H, et al. Structural basis for hijacking CBF-beta and CUL5 E3 ligase complex by HIV-1 Vif. Nature 2014,505:229-233.

28. Ichimura Y, Kumanomidou T, Sou YS, Mizushima T, Ezaki J, Ueno T, et al. Structural basis for sorting mechanism of p62 in selective autophagy. J Biol Chem 2008,283:22847-22857.

29. Kozakov D, Brenke R, Comeau SR, Vajda S. PIPER: an FFT-based protein docking program with pairwise potentials. Proteins 2006,65:392-406.

30. Zar JH. Biostatistical analysis. Upper Saddle River, N.J.: Prentice-Hall College Div, 3rd edition; 1996.

31. Pankiv S, Clausen TH, Lamark T, Brech A, Bruun JA, Outzen H, et al. p62/SQSTM1 binds directly to Atg8/LC3 to facilitate degradation of ubiquitinated protein aggregates by autophagy. J Biol Chem 2007,282:24131-24145.

32. Alemu EA, Lamark T, Torgersen KM, Birgisdottir AB, Larsen KB, Jain A, et al. ATG8 family proteins act as scaffolds for assembly of the ULK complex: sequence requirements for LC3interacting region (LIR) motifs. J Biol Chem 2012,287:39275-39290.

33. Satoo K, Noda NN, Kumeta H, Fujioka Y, Mizushima N, Ohsumi Y, et al. The structure of Atg4B-LC3 complex reveals the mechanism of LC3 processing and delipidation during autophagy. EMBO J 2009,28:1341-1350.

34. Zhou D, Spector SA. Human immunodeficiency virus type-1 infection inhibits autophagy. AIDS 2008,22:695-699.

35. Klionsky DJ, Abdalla FC, Abeliovich H, Abraham RT, Acevedo-Arozena A, Adeli K, et al. Guidelines for the use and interpretation of assays for monitoring autophagy. Autophagy 2012,8:445-544.

36. Kao S, Akari H, Khan MA, Dettenhofer M, Yu XF, Strebel K. Human immunodeficiency virus type 1 Vif is efficiently packaged into virions during productive but not chronic infection. $J$ Virol 2003,77:1131-1140.

37. Turner BG, Summers MF. Structural biology of HIV. J Mol Biol 1999,285:1-32.

38. Mohammadi P, Desfarges S, Bartha I, Joos B, Zangger N, Munoz M, et al. 24 hours in the life of HIV-1 in a T cell line. PLoS Pathog 2013,9:e1003161.

39. Dang $\mathrm{Y}$, Wang $\mathrm{X}$, Zhou $\mathrm{T}$, York IA, Zheng $\mathrm{YH}$. Identification of a novel WxSLVK motif in the $\mathrm{N}$ terminus of human immunodeficiency virus and simian immunodeficiency virus $V$ if that is critical for APOBEC3G and APOBEC3F neutralization. J Virol 2009,83:8544-8552.

40. He Z, Zhang W, Chen G, Xu R, Yu XF. Characterization of conserved motifs in HIV-1 Vif required for APOBEC3G and APOBEC3F interaction. J Mol Biol 2008,381:1000-1011.

41. Marin M, Rose KM, Kozak SL, Kabat D. HIV-1 Vif protein binds the editing enzyme APOBEC3G and induces its degradation. Nat Med 2003,9:1398-1403.

42. Mehle A, Wilson $\mathrm{H}$, Zhang $\mathrm{C}$, Brazier AJ, McPike M, Pery E, et al. Identification of an APOBEC3G binding site in human immunodeficiency virus type 1 Vif and inhibitors of VifAPOBEC3G binding. J Virol 2007,81:13235-13241.

43. Russell RA, Pathak VK. Identification of two distinct human immunodeficiency virus type 1 Vif determinants critical for interactions with human APOBEC3G and APOBEC3F. $J$ Virol 2007,81:8201-8210.

44. Marcsisin SR, Narute PS, Emert-Sedlak LA, Kloczewiak M, Smithgall TE, Engen JR. On the solution conformation and dynamics of the HIV-1 viral infectivity factor. J Mol Biol 2011,410:1008-1022. 
45. Sleiman D, Bernacchi S, Xavier Guerrero S, Brachet F, Larue V, Paillart JC, et al.

Characterization of RNA binding and chaperoning activities of HIV-1 Vif protein: Importance of the C-terminal unstructured tail. RNA Biol 2014,11:906-920.

46. Behrends C, Sowa ME, Gygi SP, Harper JW. Network organization of the human autophagy system. Nature 2010,466:68-76.

47. von Muhlinen N, Akutsu M, Ravenhill BJ, Foeglein A, Bloor S, Rutherford TJ, et al. LC3C, bound selectively by a noncanonical LIR motif in NDP52, is required for antibacterial autophagy. Mol Cell 2012,48:329-342.

48. Thurston TL, Ryzhakov G, Bloor S, von Muhlinen N, Randow F. The TBK1 adaptor and autophagy receptor NDP52 restricts the proliferation of ubiquitin-coated bacteria. Nat Immunol 2009,10:1215-1221.

49. Fouchier RA, Simon JH, Jaffe AB, Malim MH. Human immunodeficiency virus type 1 Vif does not influence expression or virion incorporation of gag-, pol-, and env- encoded proteins. J. Virol. 1996,70:8263-8269.

50. Munz C. Beclin-1 targeting for viral immune escape. Viruses 2011,3:1166-1178.

51. Kyei GB, Dinkins C, Davis AS, Roberts E, Singh SB, Dong C, et al. Autophagy pathway intersects with HIV-1 biosynthesis and regulates viral yields in macrophages. J Cell Biol 2009,186:255268.

52. Denizot M, Varbanov M, Espert L, Robert-Hebmann V, Sagnier S, Garcia E, et al. HIV-1 gp41 fusogenic function triggers autophagy in uninfected cells. Autophagy 2008,4:998-1008.

53. Lang T, Schaeffeler E, Bernreuther D, Bredschneider M, Wolf DH, Thumm M. Aut2p and Aut7p, two novel microtubule-associated proteins are essential for delivery of autophagic vesicles to the vacuole. EMBO J 1998,17:3597-3607.

54. Tanida I, Ueno T, Kominami E. LC3 conjugation system in mammalian autophagy. Int J Biochem Cell Biol 2004,36:2503-2518.

55. Gregoire IP, Richetta C, Meyniel-Schicklin L, Borel S, Pradezynski F, Diaz O, et al. IRGM is a common target of RNA viruses that subvert the autophagy network. PLoS Pathog 2011,7:e1002422. 


\section{Figure Legends}

Fig. 1. Vif interacts with human ATG8 family proteins. (a) GST pull-down assay using $\left[{ }^{35} \mathrm{~S}\right]$-labeled in vitro translated Myc-Vif and immobilized GST or indicated GST fusion proteins. Autoradiograph (upper panel) and Coomassie staining of immobilized proteins (CBB, lower panel) are shown. (b) GST pull-down assay in HEK cells after co-transfection of a plasmid expressing 3xFlag-Vif and a plasmid expressing GST alone or GST-LC3A, GST-LC3B or GST-LC3C in presence of MG132. (c) Immunoprecipitation (IP) of endogenous LC3 with anti-LC3 Ab in primary CD4 T cells infected or not by co-culture with HEK cells transfected by the HIV-1 molecular clone, in presence of MG132. A control without this Ab was added. (d) GST pull-down assay in HEK cells after transfection of a plasmid expressing 3xFlag-Vif, a plasmid expressing GST alone or GST-LC3 and a plasmid expressing, or not, A3G in presence of MG132. Co-precipitated proteins were detected with the indicated Abs. Data are representative of at least 2 independent experiments.

Fig. 2. The C-terminal domain of Vif interacts with LC3. (a) Sequence and crystal structure of Vif from the NL4.3WT strain where the 3 putative LIRs are highlighted (arrows). The three motifs, $\mathrm{HCCH}$ or zinc-finger, SOCS box and PPLP discussed in the text are depicted in red, magenta and green, respectively. (b) GST pull-down assay in HEK cells after co-transfection of a plasmid expressing 3xFlag-Vif or 3xFlag-Vif mutLIR1-3, where the first amino acid of each putative LIR has been mutated to alanine, and a plasmid expressing GST or GST-LC3 in presence of MG132. (c) Rigid docking pauses obtained for Vif (1-172) (grey, red and magenta) and LC3 (blue) corresponding to the most populated solution driven by hydrophobic contacts. The C-terminal G120 of LC3 is indicated. (d) Rigid docking pauses obtained for the SOCS deletion mutant of Vif (1-143) and LC3 (blue) showing a different binding with a large rotational motion. (e) Schematic representation of WT Vif and truncation mutants. (f) GST pull-down assay in HEK cells after co-transfection of a plasmid expressing 3xFlag coupled to WT Vif or to the truncated forms of Vif shown in (e), and a plasmid expressing GST or GST- 
LC3 in presence of MG132. Co-precipitated proteins were detected with the indicated Abs. Data are representative of at least 3 independent experiments.

Fig. 3. The pro-LC3 and Atg4-cleaved LC3 interact with Vif. (a) Schematic representation of pro-LC3 and pro-LC3 F80A/L82A. (b) GST pull-down assay in HEK cells after co-transfection of a plasmid expressing 3xFlag-Vif and a plasmid expressing GST, GST-LC3 or GST-LC3 F80A/L82A in the presence of MG132. (c) Schematic representation of LC3 1-120 and LC3 1-119. (d) GST pull-down assay in HEK cells after co-transfection of a plasmid expressing 3xFlag-Vif and a plasmid expressing GST alone or coupled to the LC3 forms described in the figure in the presence of MG132. (e) Anti-Flag IgG pulldown assay in HeLa cells that stably express A3G transfected with a plasmid expressing 3xFlag-Vif or 3xFlag-Vif1-99 in conditions that induce, or not, the formation of autophagosomes (starvation for $4 \mathrm{~h}$ in the presence of pepstatin A and E64d) [35] and in the presence of MG132. Co-precipitated proteins were detected with the indicated Abs. Data are representative of at least 2 independent experiments.

Fig. 4. Neosynthesized Vif inhibits autophagy during HIV-1 infection in the presence or absence of A3G (a) Jurkat cells were infected by NL4.3WT or NL4.3AVif for 4 days at $1 \mathrm{MOI}$ ( $70 \%$ of cells were infected). Then, cells were fixed, and LC3 and p24 were detected by immunofluorescence. The number of autophagosomes (LC3 dots) was counted by 3 investigators in more than 100 infected cells (p24 + cells). Infected cells containing at least 8 LC3 dots were defined as autophagic cells. Results are from at least 3 independent blind experiments. (b) H9 cells were infected by NL4.3WT or

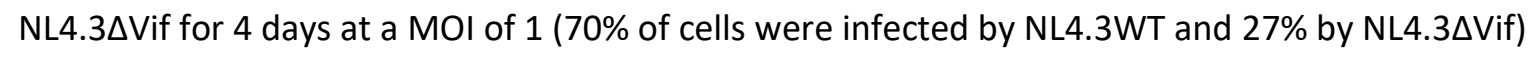
and autophagy was analyzed in infected cells as described in (a). (c) Primary CD4 T cells were infected by NL4.3WT or NL4.3AVif for 4 days ( $\mathrm{MOI}$ of 1; approximately $30 \%$ and $15 \%$ of cells, respectively, were infected). Infected cells containing at least 3 LC3 dots were defined as autophagic 
cells. (d) Primary CD4 T cells were infected for 4 days, and chloroquine was added for 3 h before cell fixation. (e) HEK-CD4-CXCR4 cells were transfected with a plasmid expressing GFP-LC3. 24h later, cells were co-cultured for 1 day with Jurkat cells that have been previously infected by NL4.3WT or NL4.3AVif ( $50 \%$ of cells were infected), MoltX4 or 8 E5 cells. Then, HEK-CD4-CXCR4 cells were fixed and labeled using an anti-p24 Ab. Autophagic cells were defined as cells containing at least 5 LC3 dots. More than 100 infected cells were counted by 3 investigators and results are from at least 3 independent blind experiments. Scale bars $=10 \mu \mathrm{m}$.

Fig. 5. Vif C-terminal domain inhibits autophagy through binding to LC3. (a) HEK-CD4-CXCR4 cells were transfected with a plasmid expressing GFP-LC3. (b) HEK-CD4-CXCR4 cells were transfected with a plasmid expressing GFP-LC3 and a plasmid expressing 3xFlag-Vif. (c) HEK-CD4-CXCR4 cells were transfected with a plasmid expressing GFP-LC3 and a plasmid expressing 3xFlag-Vif1-99. Four hours later, all the cells $(a, b, c)$ were infected for 2 days by free NL4.3AVif or NL4.3WT at a MOI of 1 . Then, cells were fixed and labeled using an anti-p24 Ab. Autophagic cells are defined as cells containing at least 5 green LC3 positive spots. More than 100 infected cells were counted by 3 investigators and results are from at least 3 independent blind experiments. Scale bars $=10 \mu \mathrm{m}$.

\section{Supplemental figures}

Supplemental Fig. 1. Autophagy is inhibited by Vif after $\mathbf{1 1}$ days of infection (a) H9 cells were infected by NL4.3WT or NL4.3AVif for 11 days at a MOI of 1 . Then, cells were fixed and autophagy was analyzed as described in Fig.4. (b) Chloroquine was added to infected H9 cells for 3 h before cell fixation. Scale bars $=10 \mu \mathrm{m}$.

\section{Supplemental Fig.2. Vif inhibits autophagy in HIV-infected Jurkat cells and in HIV-transfected HEK}

cells. (a) LC3-II expression was analyzed in Jurkat cells infected by NL4.3WT or NL4.3AVif for 3 days at a MOI of 1. Fold induction was calculated as the intensity of the LC3-II immunoblot obtained in cells 
infected by NL4.3WT compared to cells infected by NL4.3AVif. Values were normalized to those obtained with anti-GAPDH Ab. (b) HEK cells were transfected with the molecular clone pNL4.3WT or pNL4.3AVif for $24 \mathrm{~h}$. LC3-II expression was analyzed by western blotting. Fold induction was calculated as the intensity of the LC3-II immunoblot obtained in cells transfected by pNL4.3WT compared to cells transfected by pNL4.3AVif. Values were normalized to those obtained with antiGAPDH Ab. Data are representative of at least 5 independent experiments.

\section{Supplemental Fig. 3. Vif inhibits autophagy induced by drugs when ectopically expressed in HEK} cells. (a) HEK cells were transfected with the plasmid expressing mCherryVif. One day later, $20 \mu \mathrm{M}$ MG132 and $2 \mu \mathrm{M}$ Torin were added for $4 \mathrm{~h}$. Cells were then fixed, and endogenous LC3 was labeled using the anti-LC3 Ab as described in the section Materials and Methods. Results are from at least 2 independent blind experiments. (b) Autophagy was induced by starvation (EBSS for $3 \mathrm{~h}$ ) in HEK cells transfected with either a plasmid expressing Flag or Flag-Vif, in presence or absence of anti-proteases (pepstatin A and Ed64 at $10 \mu \mathrm{g} / \mathrm{mL}$ ). LC3-II expression was analyzed by western blotting and was normalized to the GAPDH expression level. Data are representative of 3 independent experiments.

\section{Supplemental Fig. 4. Vif increases intracellular p24 expression independently of the presence of}

A3G. (a) HEK-CD4-CXCR4 cells were transfected with the same amount of pNL4.3WT or pNL4.3AVif. 24h later, these cells were co-cultured for 3 days with Jurkat cells. The percentage of infected CD4 T cells was analyzed by FACS. (b) p24 was measured in lysates of Jurkat cells infected as described in (a) using a p24 antigen capture ELISA (Beckman Coulter). (c) SupT1cells infected by co-culture as described in (a) were enriched by limiting dilution cloning and expression of p55Gag and p24 proteins was analyzed by Western blotting using the anti-Gag Ab.

\section{Supplemental Fig.5. LC3 cannot bind to the SOCS box-like motif when the EloB/C complex is bound} to Vif. (a) Binding prediction of LC3 (blue) on Vif (grey, red and magenta) obtained by rigid docking as 
shown in Figure 2c. (b) Crystal structure of the complex between Vif and EloB/C (both Elongin chains are shown in orange and Cullin 5 chains are depicted in tan ribbon). 

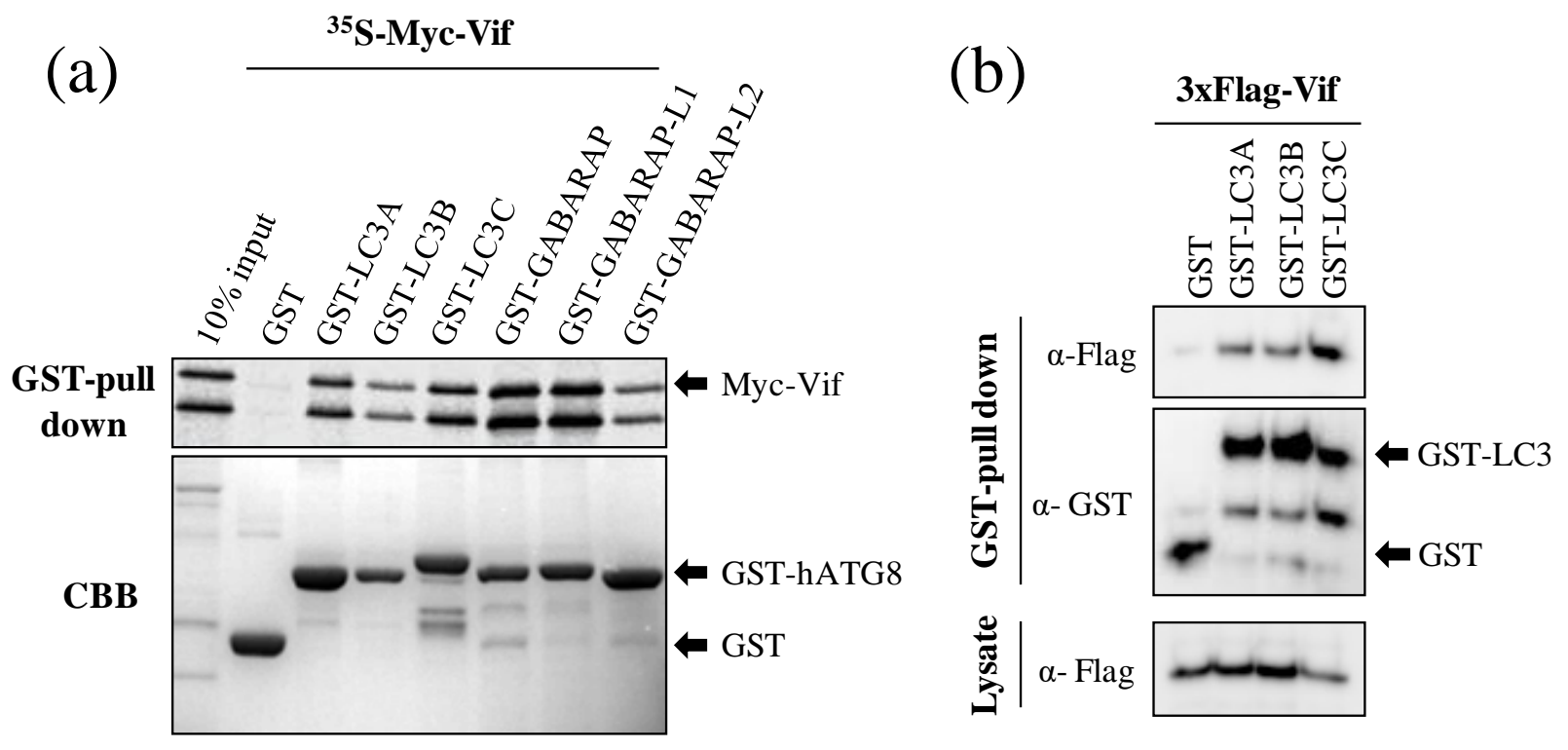

(c)

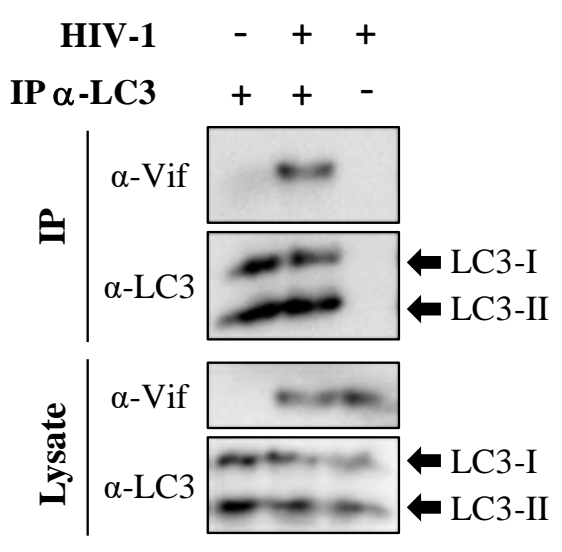

(d) $\quad \frac{\text { 3xFlag-Vif }}{\mathbf{A} 3 \mathbf{G}}$

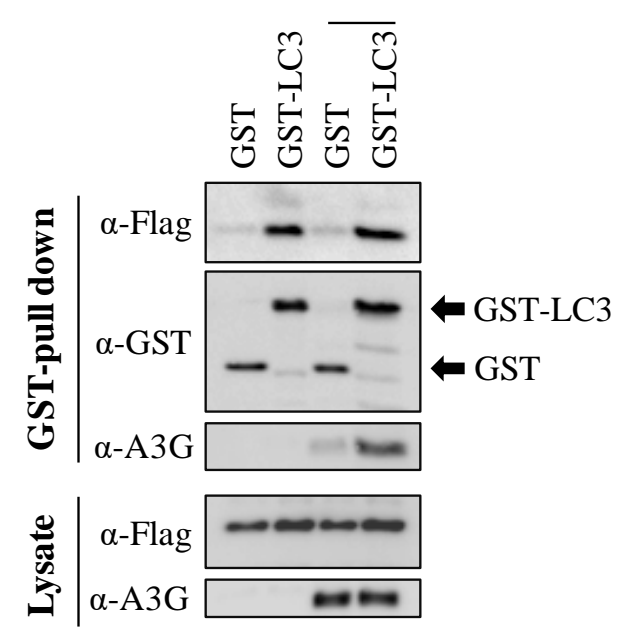


(a)

NRWQVMIV WQVDRMRINT WKRLVKHHMY ISRKAKDWFY RHHYESTNPK ISSEVHIPLG DAKLVITTYW GLHTGERDWH LGQGVSIEWR KKRYSTQVDP DLADQLIHLH YFDCFSESAI RNTLLGRIVS PRCEYQAGHN KVGSLQYLAI AALIKPKQIK PPLPSVRKLT EDRWNKPQKT KGHRGSHTMN GH

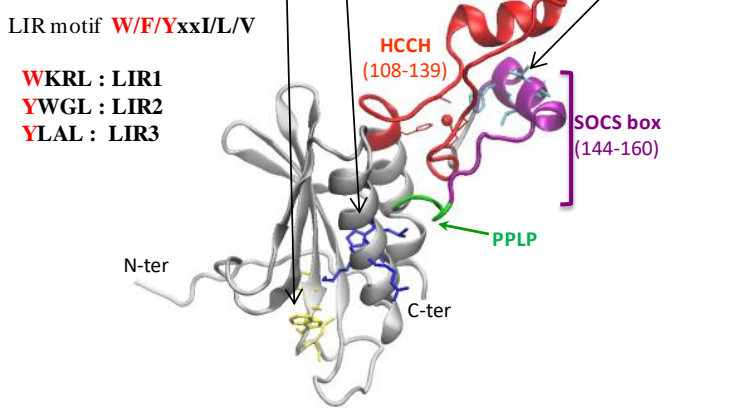

(c)

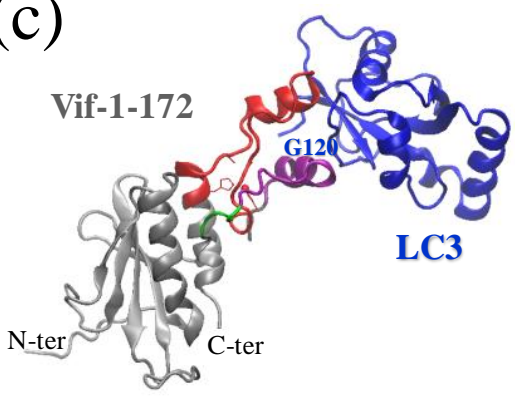

(e)

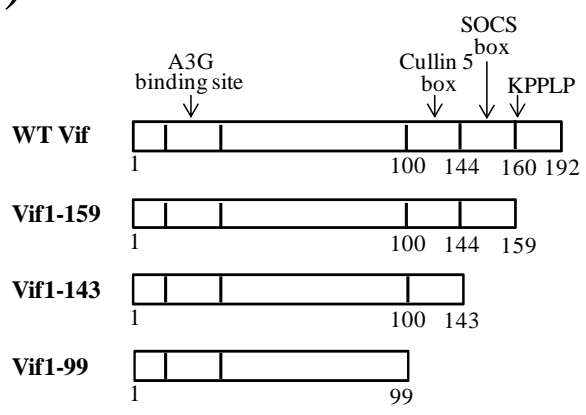

(b)

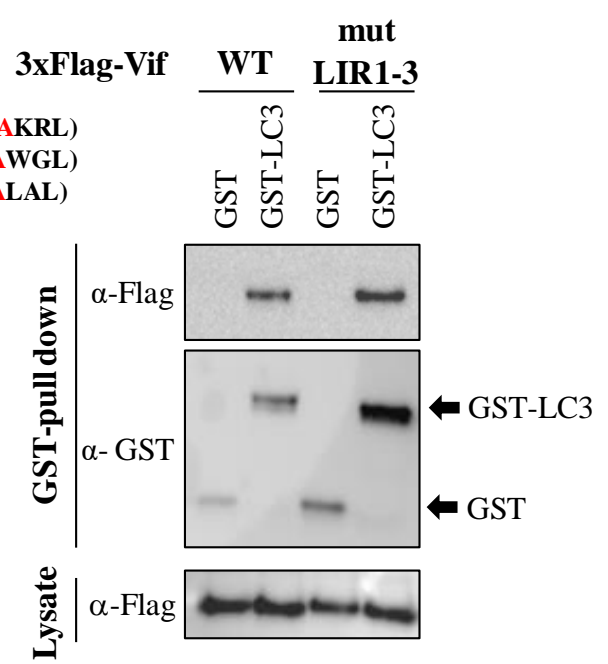

(d)

mut LIR1 $(W \rightarrow$ AKRL $)$
mut LIR2 $($ Y $\rightarrow$ AWGL $)$

mut LIR3 $(\mathrm{Y} \rightarrow$ ALAL $)$

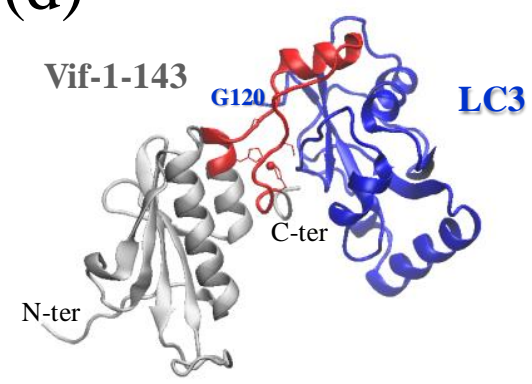

(f)

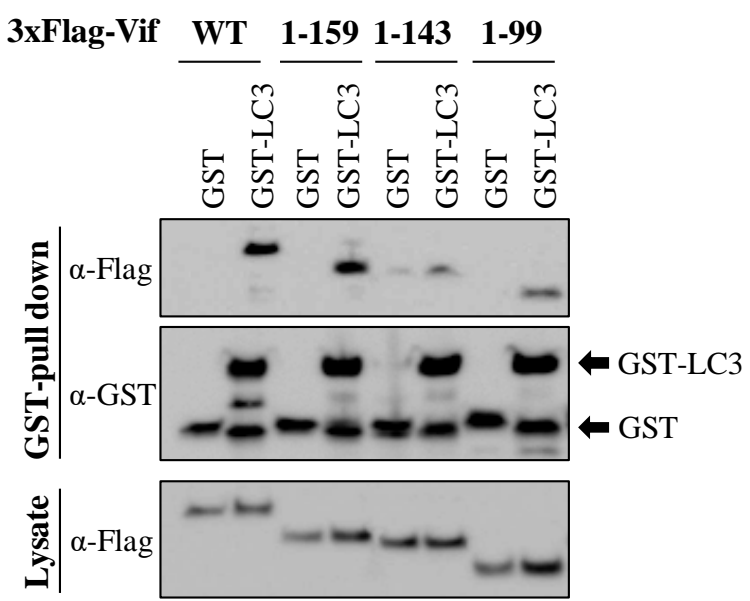


(a)

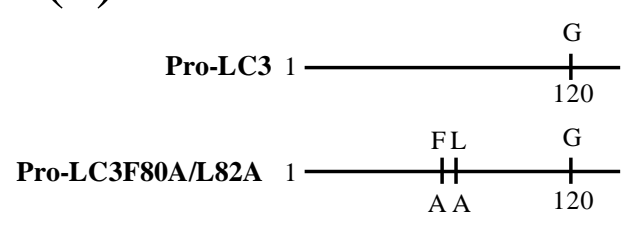

(c)

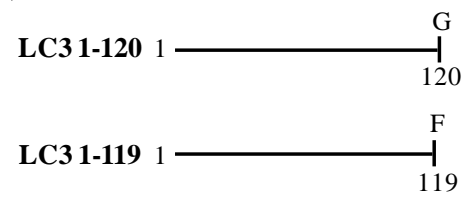

(b)
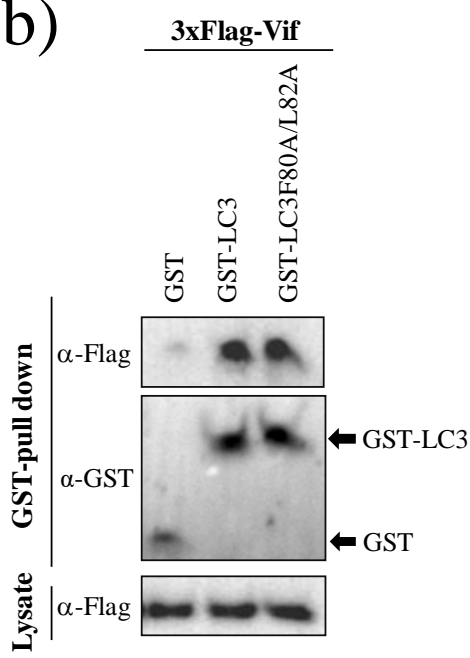

(d)

$\frac{\text { 3xFlag-Vif }}{\circ 9}$

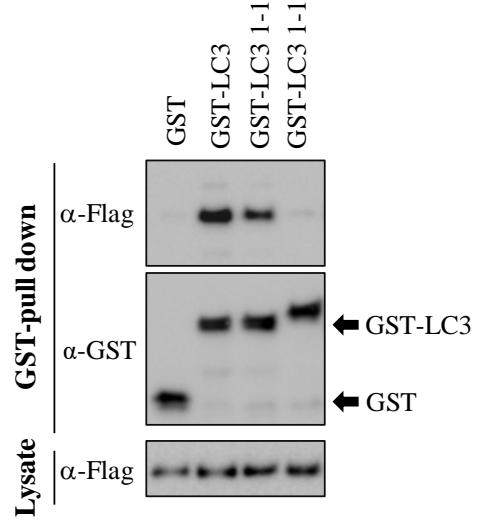

(e)
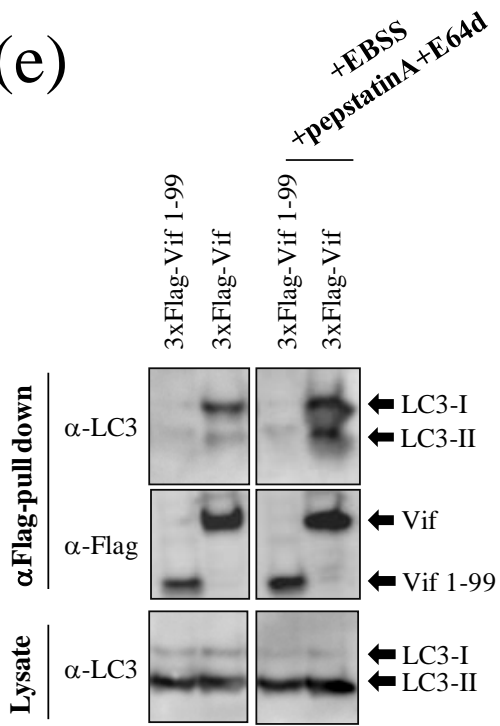


\section{Figure 4}

(a)

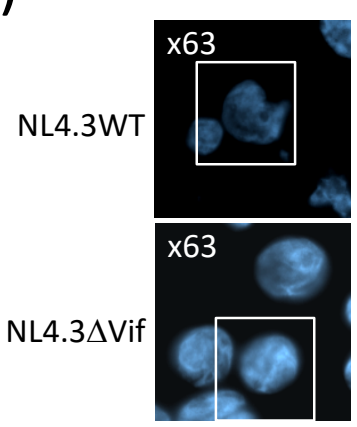

Dapi

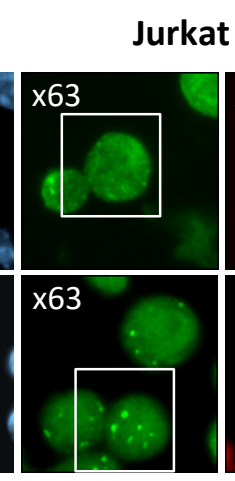

LC3

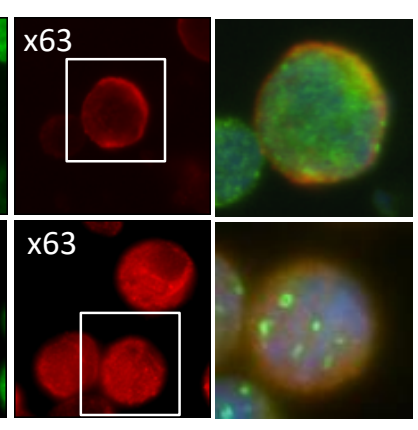

p24

Overlay

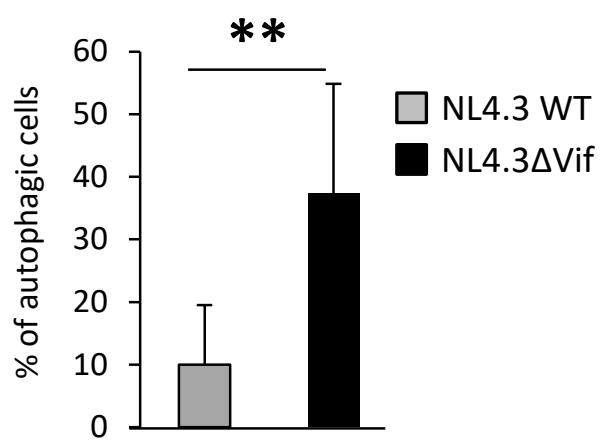

(b)

NL4.3WT

NL4.3 $\Delta$ Vif

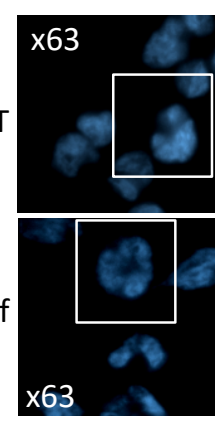

Dapi

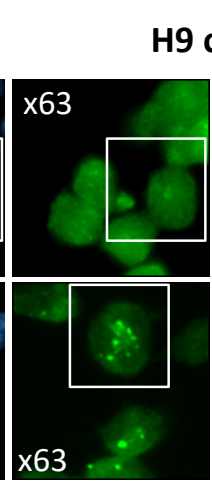

LC3

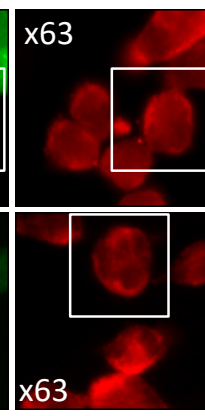

p24

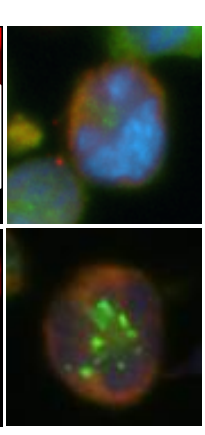

Overlay

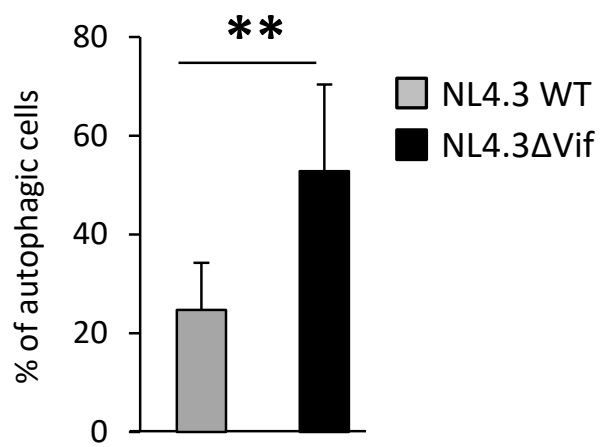

(C)

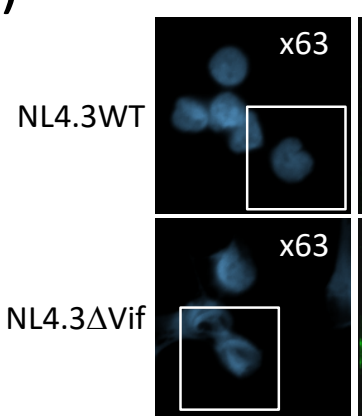

Dapi

Primary CD4 T cells
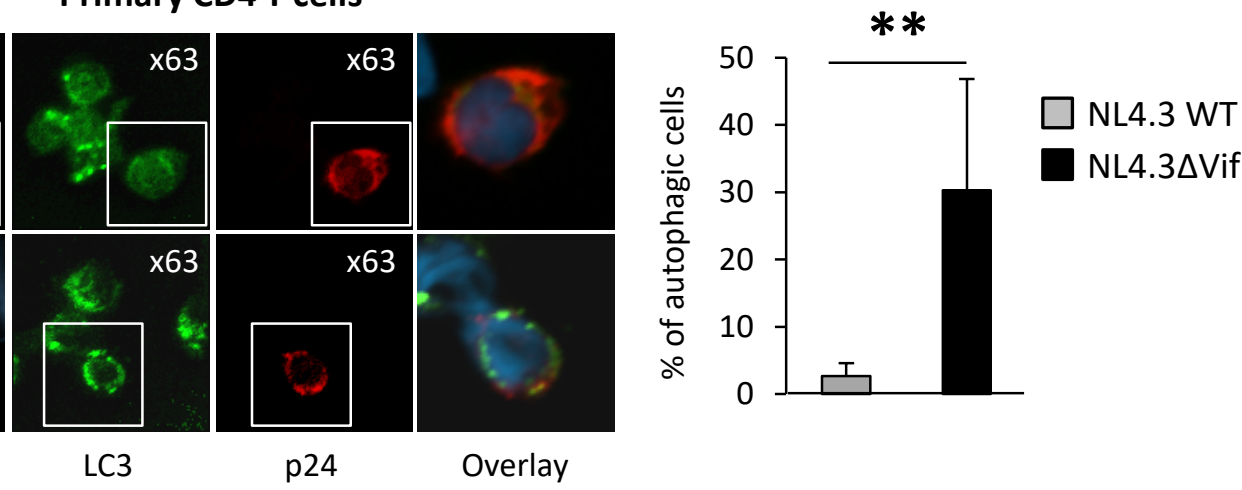

(d)

NL4.3WT

Dapi

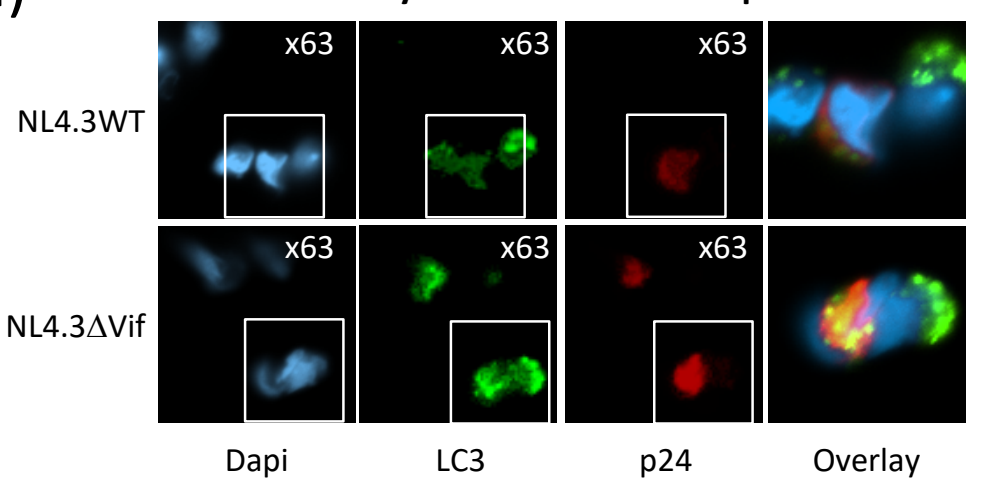

Primary CD4 T cells + chloroquine

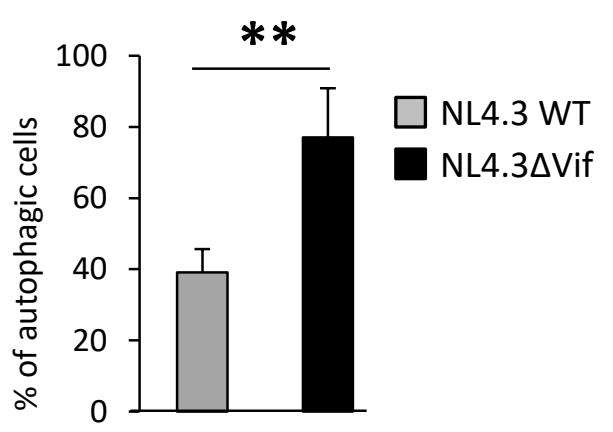


(e)

HEK CD4-CXCR4

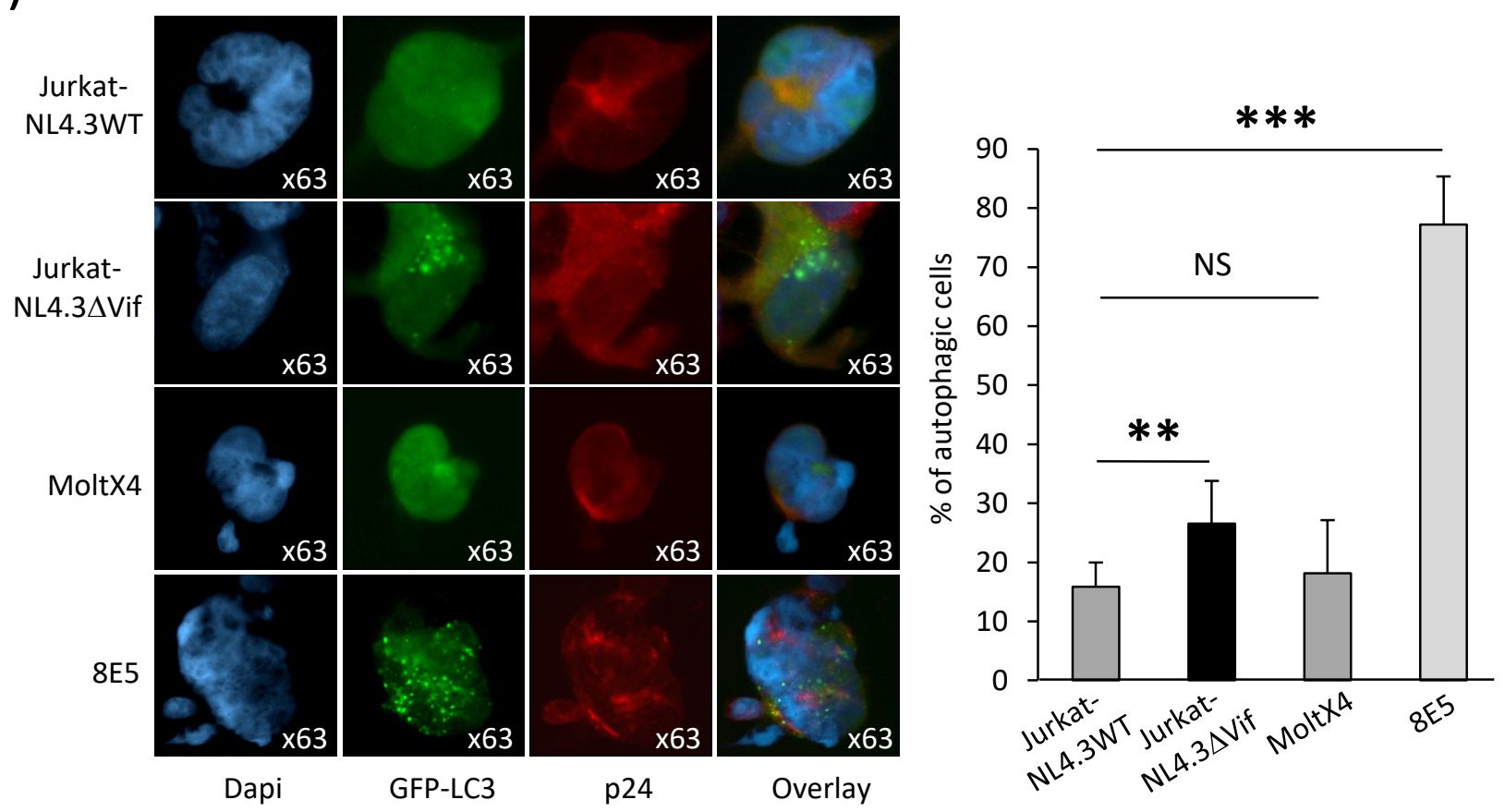




\section{Figure 5}

(a)

No complementation

NL4.3WT
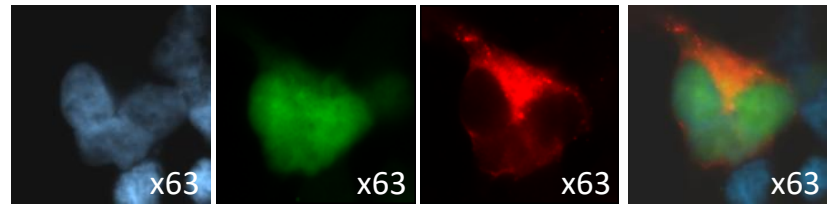

NL4.3 $\Delta$ Vif
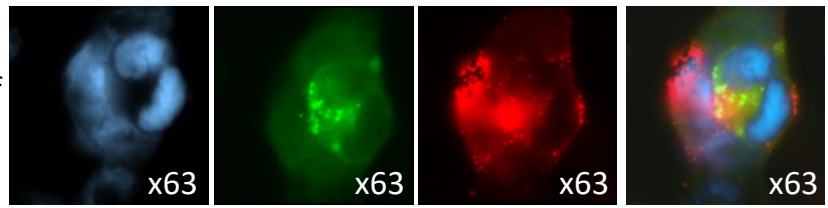

(b)

WT Vif complementation

NL4.3WT
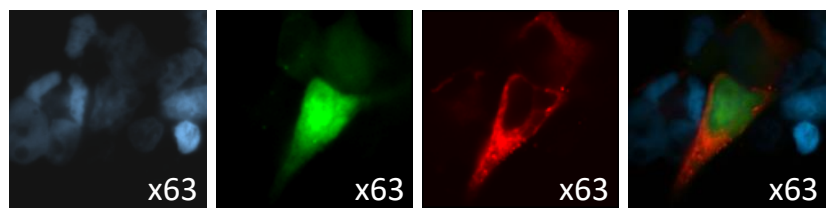

NL4.3 $\Delta$ Vif
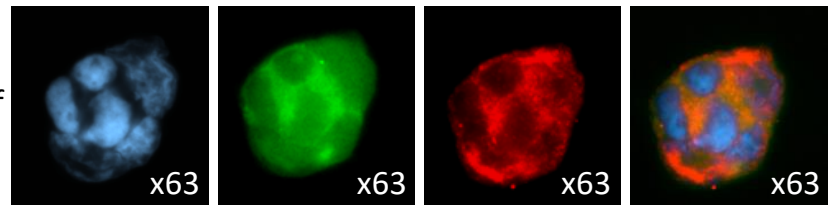

(c)

Vif 1-99 complementation

NL4.3WT
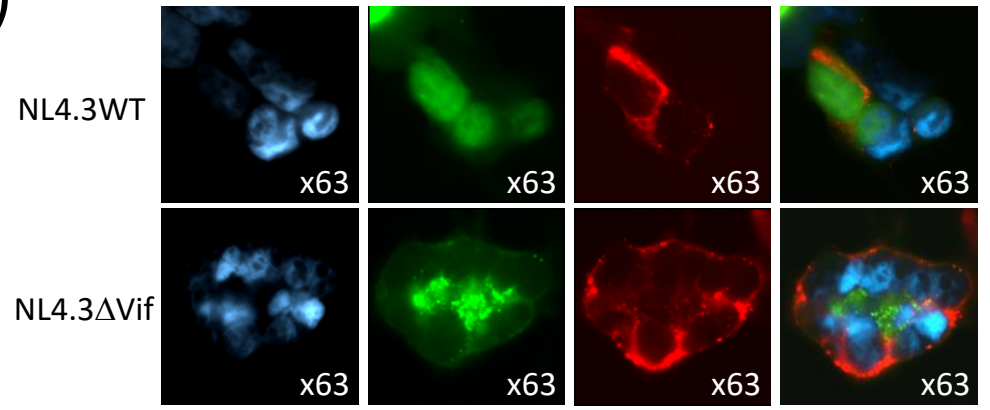

Dapi

GFP-LC3

p24

Overlay

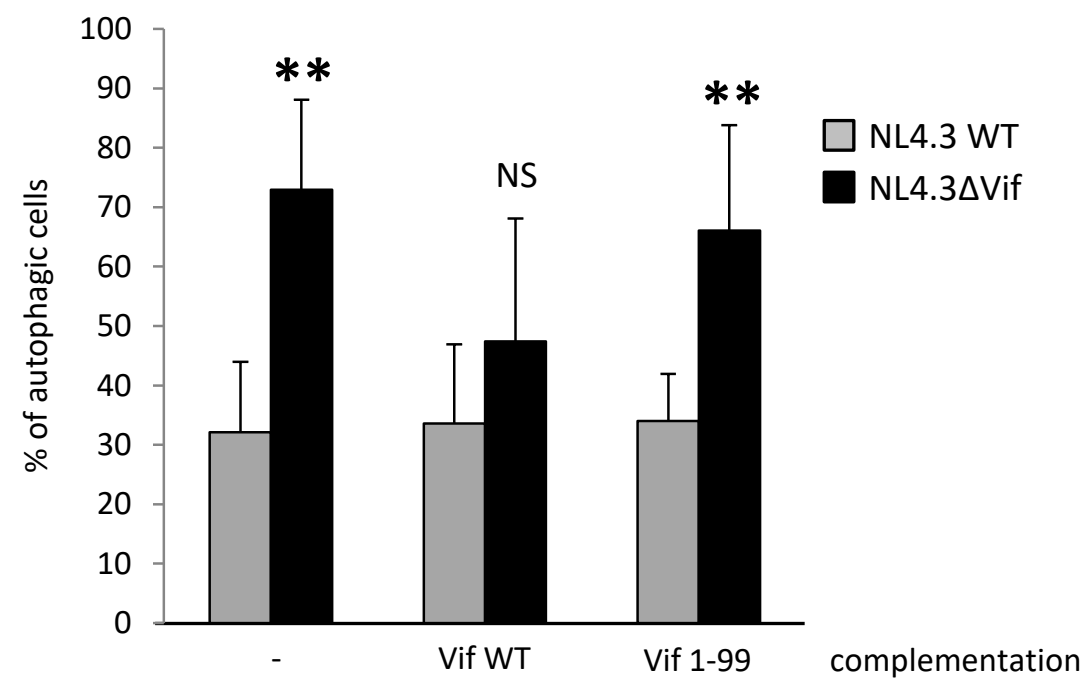




\section{Supplemental figure 1}
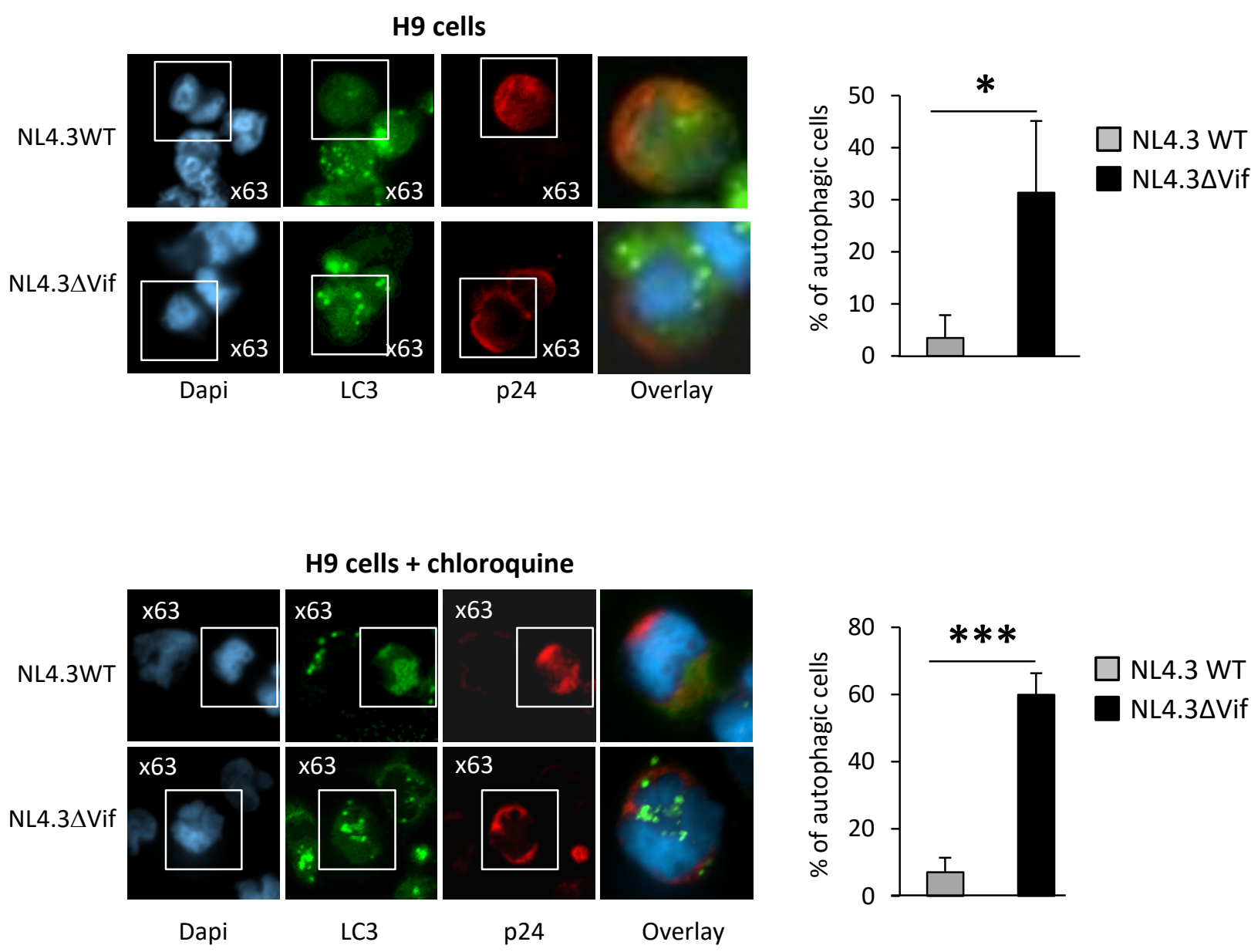
(a)

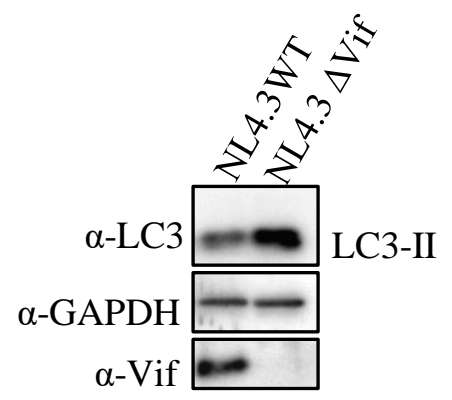

LC3-II/GAPDH 12.7 (b)

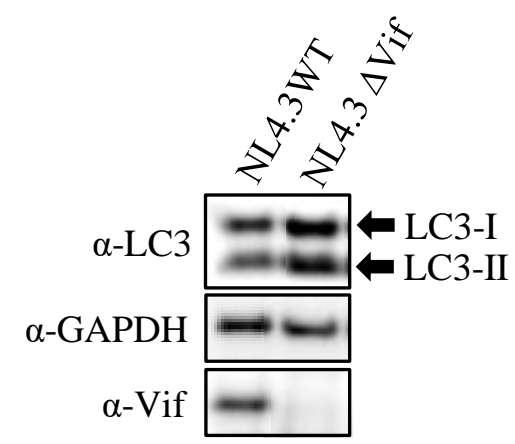

LC3-II/GAPDH $\quad \mathbf{1} \quad \mathbf{2 . 7}$ 


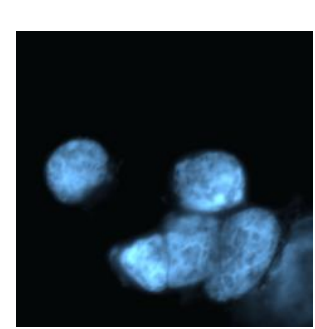

Dapi

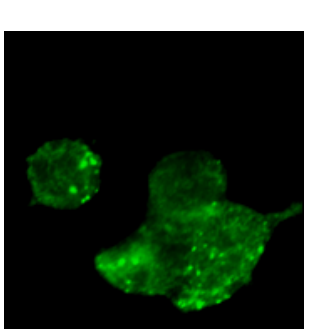

LC3

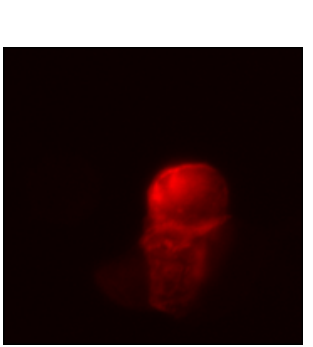

mCherryVif

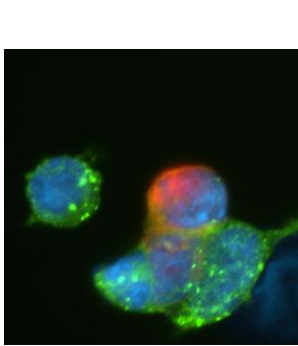

Overlay

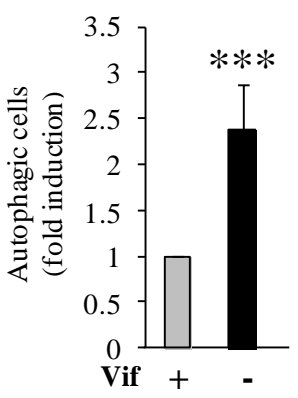




\section{Supplemental Figure 4}

(a)

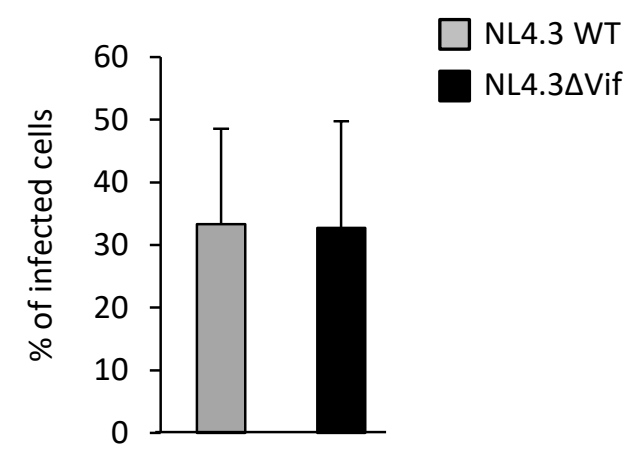

(b)

(c)

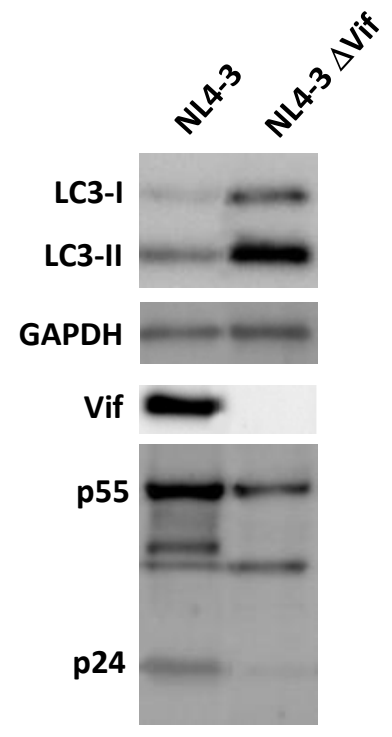


(a)

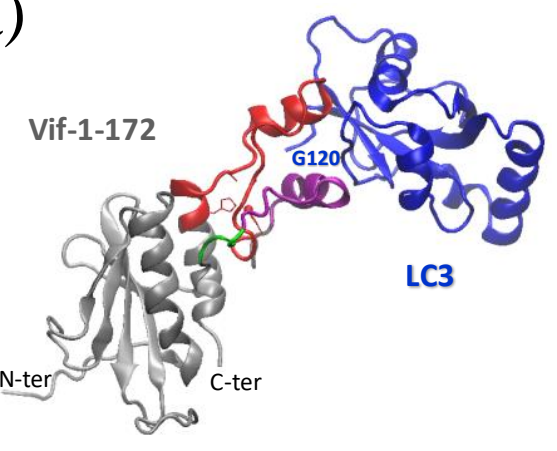

(b)

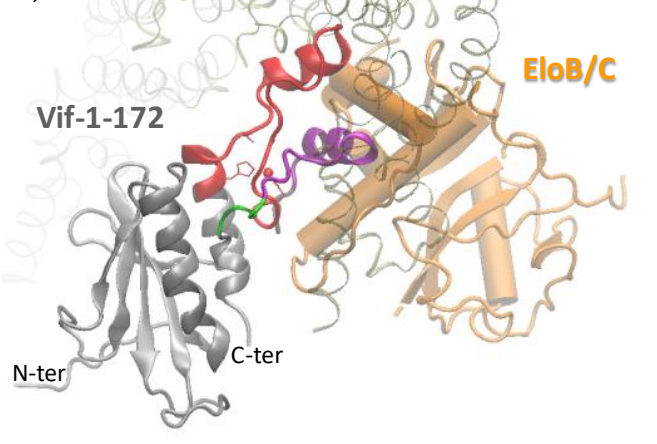

